\title{
Cardiotocography in a perinatal armamentarium: boon or bane?
}

\author{
Ambily Anu Xavier, Deeksha Pandey*, Luvdeep Dogra, Leslie Edward Lewis
}

KMC Manipal, Manipal University, Manipal - 576104, India

Received: 19 October 2015

Accepted: 23 November 2015

*Correspondence:

Dr. Deeksha Pandey,

E-mail: ardentpreety@gmail.com

Copyright: (c) the author(s), publisher and licensee Medip Academy. This is an open-access article distributed under the terms of the Creative Commons Attribution Non-Commercial License, which permits unrestricted non-commercial use, distribution, and reproduction in any medium, provided the original work is properly cited.

\section{ABSTRACT}

Background: As electronic foetal monitoring (EFM) is becoming commoner in obstetric armamentarium, so are caesarean deliveries. Present study was conducted with an aim to find out correlation between cardiotocography (CTG) findings, intraoperative findings, and perinatal outcome in subjects who underwent emergency caesarean deliveries. We also intended to estimate the sensitivity, specificity and positive predictive value of CTG in diagnosing foetal distress.

Methods: A total of 271 women were included. Based on the CTG findings, the patients were divided into two groups: A) Case group: comprising subjects with Category II (suspicious) and Category III (pathological) CTG tracings; B) Control group: comprising subjects with Category I CTG tracings.

Results: We found that $90.5 \%$ women with suspicious and pathological CTG (cases) undergoing emergency caesarean had one or the other abnormal per-operative findings that might cause foetal distress or CTG abnormality. Around a third (33.7\%) with abnormal CTG had normal per-operative findings and good neonatal outcome suggesting false positivity. APGAR score of less than 5 at 5 minutes was seen in $17.9 \%$ of patients with pathological CTG. The sensitivity, specificity, positive predictive value and negative predictive value of CTG for prediction of an abnormal perinatal outcome were found to be $90.5,66.3,44.9$, and 95.8 respectively.

Conclusions: CTG should only be used as a screening tool for monitoring of foetal status during labour. It is worth remembering that normal CTG is more predictive of normal outcomes than abnormal CTG regarding abnormal outcomes.

Keywords: CTG, Emergency caesarean, Perinatal outcome

\section{INTRODUCTION}

Foetal heart rate (FHR) monitoring is a part of intrapartum surveillance of foetus. It is of two types intermittent auscultation (IA) and electronic foetal heart rate monitoring (EFM). EFM when used during labour, comprises of a continuous record of the FHR pattern over a desired length of time, on a two - channel chart, with FHR on the upper channel, and frequency, duration and amplitude of uterine contractions on the lower channel constituting cardiotocography (CTG). CTG was introduced into obstetrical practice in the 1960s, primarily to monitor complicated pregnancies. ${ }^{1,2}$ Although the original purpose does not apply to all pregnancies, use of EFM in labouring women has been on the rise, despite the lack of clinical trials to support its efficacy in reducing perinatal adverse outcomes. ${ }^{3}$

As EFM is becoming commoner, so are caesarean deliveries, many of which might be unnecessary owing to misinterpretation (subjective errors) or high false positive rates. The estimated caesarean delivery rate in India in the year 1998 was $7.1 \%$ with an average annual increase of $16.7 \%$, which is one of the highest among the countries of South East Asia region.4 Interestingly foetal distress accounts for around a third (32\%) of all caesareans. ${ }^{5}$ There has to be a delicate hairline balance between distinguishing a foetus that exhibits a stress- 
response to labour, from the one that shows a 'distress' or hypoxic response. Failure to do so increases unnecessary intervention in the former, while increasing the morbidity and mortality in latter.

Furthermore, caesarean deliveries may be associated with maternal complications like damage to adjacent structures intra-operatively, haemorrhage, risk of infections, tendency for repeat caesarean, rupture of uterus and incisional hernia later. Also, it adds on to the financial burden in the developing countries.

Present study was conducted with an aim to find out correlation between CTG findings, intraoperative findings, and perinatal outcome in subjects who underwent emergency caesarean deliveries. Indirectly we wanted to find out justification for a caesarean delivery which was based entirely on a CTG trace. We also intended to estimate the sensitivity, specificity and positive predictive value of CTG in diagnosing true foetal distress/birth asphyxia.

\section{METHODS}

This prospective study was carried out in the labour ward at a tertiary care centre during a one year (1st June 2012 to 31st May 2013) period. The study protocol was approved by institutional ethics committee. All enrolled women provided written informed consent for participation. Consent was obtained from all women with normal singleton, cephalic-presenting foetuses between 37 to 41 weeks period of gestation who were admitted in the labour room during the study period. During this period a total of 427 pregnant women underwent emergency caesareans. Out of these 143 caesareans were done for either preterm, twins, non-cephalic or complicated pregnancies and thus were excluded. Only 284 women fulfilled our inclusion criteria and were recruited for the study.

Gestational age was determined on the basis of the last menstrual period and a reliable menstrual history, and/or an ultrasound examination before 16 week's gestation. Both intact and ruptured membranes were included in this study.

As part of our labour theatre protocol, all patients had continuous EFM with Avalon Foetal Monitor FM20 (PHILIPS). All CTG tracings were interpreted prospectively with the help of a senior consultant in Obstetrics. The variables used for analysis were base line FHR, beat-to-beat variability, number of accelerations and number of decelerations in a 20-minute time interval. That 20-minute CTG, which was done immediately prior to the caesarean was taken into consideration.

During caesarean section, meconium staining of liquor, loops of cord around neck, true knots in cord, whether infant cried after delivery, and any other atypical finding were noted. Also, perinatal outcome was recorded under the headings of birth weight, and APGAR score. Demographic details and indication for caesarean section for the study subjects were simultaneously recorded.

Based on the CTG findings, the patients were divided into two groups: A) Case group: comprising subjects with Category II (suspicious) and Category III (pathological) CTG tracings; B) Control group: comprising subjects with Category I CTG tracings. These women which comprised the control group had caesarean because of indications other than foetal distress. The categorization of CTG tracings was done according to National Institute of Clinical Excellence (NICE) guidelines. (NICE clinical guideline 55, September 2007).

Data Analysis: All calculations were made using SPSS 21.0 IBM Statistics released August 2012 (IBM Corporation 1, New Orchard Road, Armonk, New York, 10504-1722 U.S.). Categorical variables were compared using Fisher exact test (2 sided). Significance was set at $\mathrm{P}<0.05$. Later based on perinatal outcome sensitivity, specificity and predictive values of CTG was also calculated using the standard formulas.

\section{RESULTS}

As mentioned earlier 284 women who fulfilled our inclusion criteria and were recruited for the study. Out of these 13 women were excluded from the study as 9 caesareans were performed under general anaesthesia as per the anaesthesiologist's decision and for four cases certain data was found to be incomplete/missing during the final analysis. So for the final analysis 271 women were included. Among these 144 women fell in the control group while 127 women (99 suspicious CTG and 28 pathological CTG) comprised the case group. (Figure 1)

Mean age group of women in control and test group was 24.8 and 24.8 years respectively. Medical and obstetrics complications was comparable in both the groups. Even the period of gestation at the time of delivery was found to be matching (control: 38.59 versus cases: 38.46 weeks).

On comparing the overall abnormal per-operative finding that might cause foetal distress, CTG abnormality were found to be twenty times less in the control group $(90.5 \%$ abnormal per-operative findings in cases versus $4.2 \%$ in controls). There was a positive correlation with abnormal CTG and abnormal per-operative findings in $90.5 \%$ cases. Similarly there was a negative correlation in $66.3 \%$ of controls among the two variables. Detailed description of these abnormal per-operative findings in relation to the various categories of CTG is tabulated in table 1 . While $138(95.8 \%)$ women in the control group had no abnormal per-operative finding, only $70(55 \%)$ among the cases [60 women $(60.6 \%)$ with suspicious CTG and 10 women $(35.7 \%)$ with pathological CTG] had normal peroperative findings. Meconium stained amniotic fluid 
(MSAF) was the most frequent per-operative abnormal finding which could be associated with foetal distress. The incidence of MSAF was significantly higher in the cases. Both the subgroups in cases had high frequency of MSAF (suspicious: 30.3\%; pathological: $57.1 \%$ - CTG groups) with statistically significant higher incidence in the pathological CTG sub-group. There were 7 cases of cord around neck and all these were in the suspicious
CTG sub-group of cases. True knots were found two in each of suspicious and pathological CTG groups. Neither true knot nor cord around neck was found in the control group. (Table 1) It was apparent that CTG findings overall had a positive correlation with the per-operative findings which are indirect predictors of foetal distress/birth asphyxia.

Table 1: Comparison of abnormal pre-operative findings among cases and controls.

\begin{tabular}{|c|c|c|c|c|c|c|c|c|}
\hline & & \multicolumn{6}{|c|}{ CTG abnormalities } & \multirow{4}{*}{ p-value } \\
\hline & & Con & & $\mathrm{Ca}$ & & & & \\
\hline & & \multicolumn{2}{|c|}{ Normal $=144$} & \multicolumn{2}{|c|}{ Suspicious $=99$} & \multicolumn{2}{|c|}{ Pathological $=28$} & \\
\hline \multirow{5}{*}{$\begin{array}{l}\text { Per- } \\
\text { operative } \\
\text { findings }\end{array}$} & & $\mathrm{N}$ & $\%$ & $\mathrm{~N}$ & $\%$ & $\mathrm{~N}$ & $\%$ & \\
\hline & $\begin{array}{l}\text { Meconium } \\
\text { stained } \\
\text { amniotic } \\
\text { fluid }\end{array}$ & 6 & $4.2 \%$ & 30 & $30.3 \%$ & 16 & $57.1 \%$ & \multirow[t]{4}{*}{$<0.05$} \\
\hline & Nuchal cord & 0 & $0 \%$ & 7 & $7.1 \%$ & 0 & $.0 \%$ & \\
\hline & True knot & 0 & $0 \%$ & 2 & $2.0 \%$ & 2 & $7.1 \%$ & \\
\hline & Normal & 138 & $95.8 \%$ & 60 & $60.6 \%$ & 10 & $35.7 \%$ & \\
\hline
\end{tabular}

Table 2: Perinatal outcome based on birth-weight and APGAR scores among the newborns in cases and controls.

\begin{tabular}{|c|c|c|c|c|c|c|c|c|}
\hline \multicolumn{8}{|c|}{ CTG abnormalities } & \multirow{4}{*}{ p-value } \\
\hline & & Cont & & $\mathrm{Ca}$ & & & & \\
\hline & & Norr & & $\mathrm{Sus}$ & & Pat & & \\
\hline \multirow{2}{*}{$\begin{array}{l}\text { Perinatal } \\
\text { outcome } \\
\text { Birth } \\
\text { weight }\end{array}$} & & $\mathrm{N}$ & $\%$ & $\mathrm{~N}$ & $\%$ & $\mathrm{~N}$ & $\%$ & \\
\hline & $\begin{array}{l}\text { Birth weight } \\
<2.5\end{array}$ & 8 & 5.6 & 15 & 15.2 & 3 & 10.7 & \multirow[t]{2}{*}{$<0.05$} \\
\hline \multirow{3}{*}{$\begin{array}{l}\text { Perinatal } \\
\text { outcome } \\
\text { APGAR } \\
\text { score }\end{array}$} & $\begin{array}{l}\text { Birth } \\
\text { weight }>2.5\end{array}$ & 136 & 94.4 & 84 & 84.8 & 25 & 89.3 & \\
\hline & Apgar $<7$ & 0 & - & 1 & 1 & 5 & 17.9 & \multirow{2}{*}{$<0.05$} \\
\hline & Apgar $\geq 7$ & 144 & 100 & 98 & 99 & 23 & 82.1 & \\
\hline
\end{tabular}

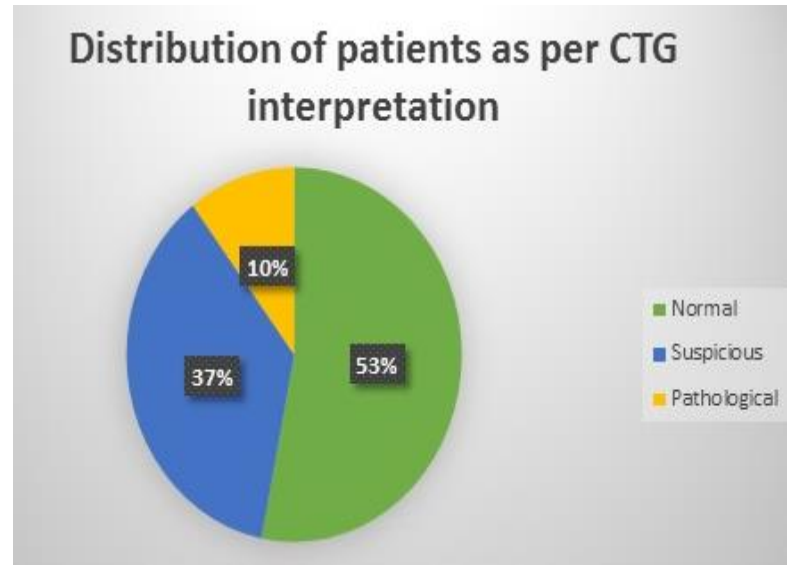

Figure 1: Pie diagram showing distribution of cases and controls in our study population.
On comparing the birth weight of the new born babies (irrespective of gestational age) $94.4 \%$ weighed above $2.5 \mathrm{~kg}$ in the control group as compared to $87.1 \%$ in the cases (Table 2). Interestingly all babies who had normal CTG preoperatively had APGAR score of more than 5 (fairly good APGAR score) at 5 minute after birth. Ten new-borns $(6.9 \%)$ from the control group had to be admitted in NICU. However in none of them birth asphyxia was the cause. Two neonates were admitted because of presence of MSAF per operatively for observation, two had asymptomatic hypoglycaemia (one born to mother with gestational diabetes), one had respiratory distress, while two were admitted because of neonatal jaundice. Three babies in this group were admitted in NICU because of some rare diagnoses. These included tuberous sclerosis, multiple cardiac rhabdomyoma, and subependymal nodules in one, congenital cystic adenomatoid malformation of right lung 
in another and high anorectal malformation, ventricular septal defect in the third one.

Only 6 babies had APGAR of less than 5, in which 5 were in the pathological CTG subgroup, while 1 was in the suspicious CTG group. In the test group overall $11 \%$ $(n=14)$ neonates required NICU admission. Nine out of these 14 had birth asphyxia (64.2\%). Fortunately only three of these nine $(33 \%)$ developed hypoxic ischemic encephalopathy. All of these three were in the pathological CTG group. Two of these neonates succumbed to death within 24 hours. There was one more death in this group but in that case baby had congenital diaphragmatic hernia.

Finally the sensitivity, specificity, positive predictive value and negative predictive value of CTG for prediction of an abnormal perinatal outcome (which is the real indicator of a distressed foetus) was found to be 90.5, $66.3,44.9$, and 95.8 respectively.

\section{DISCUSSION}

In our study we found that most of the women with suspicious and pathological CTG (cases) undergoing emergency caesarean had one or the other abnormal peroperative findings that might cause foetal distress or CTG abnormality. Around a third (33.7\%) with abnormal CTG had normal per-operative findings and good neonatal outcome suggesting false positivity. APGAR score of less than 5 was seen in $17.9 \%$ of patients with pathological CTG. Out of which two new born babies succumbed within 24 hours of delivery.

The usage of CTG in modern obstetrical care has exceeded far beyond monitoring only complicated pregnancies (for what it was initially started for) even before trials have proved its efficacy in significantly reducing perinatal adverse outcomes. Cochrane metaanalysis, last updated in 2006, compared 13 randomized trials of continuous EFM with IA and confirmed higher caesarean section rates but no improvement in Apgar scores, perinatal mortality and rates of cerebral palsy in infants born to women with continuous EFM. ${ }^{6}$ Way back in 1989 itself Grant et al had demonstrated that EFM is associated with a $50 \%$ reduction in the incidence of early neonatal seizures, but there were no differences between the EFM and IA groups in the rate of cerebral palsy when infants with newborn seizures were re-examined at 4 years of age. ${ }^{7}$

Even a more recent cochrane meta-analysis by Grivell RM et $\mathrm{al}^{8}{ }^{8}$ which included 6 studies (2105 women at increased risk of complications), comparison of traditional CTG versus no CTG showed no significant difference in perinatal mortality.

Though CTG seems to be a good screening tool with a sensitivity of 90.5 in our study its specificity is low (66.3). We may have a protocol to screen women with
CTG, but before planning for active intervention we should confirm the CTG abnormalities. In a very recent study Ropacka-Lesiak et al recommended that cerebroumbilical ratio ( $\mathrm{C} / \mathrm{U}$ ratio) should be used as a first-line test in the prediction of abnormal CTG recordings in uncomplicated pregnancies. ${ }^{9}$ However the feasibility and cost effectiveness of performing CTG for all uncomplicated pregnancies as a screening tool has to be assessed.

As far as predictability of CTG is concerned a study was conducted in Pakistan to find out the predictive value of CTG in terms of foetal acid base status at birth in women undergoing emergency caesarean section for a suboptimal CTG trace. They concluded that suspicious CTG trace has low predictive value while a pathological CTG is highly predictive of foetal acidosis at birth warranting immediate intervention. $^{10}$

Our study showed a very high negative predictive value of CTG (i.e. 95.8), which means that if CTG is normal we can be sure of foetal well-being to a certain extent. Similarly a study from Bangladesh found the CTG sensitivity of $87 \%$, specificity of $66 \%$, positive predictive value $54 \%$ and negative predictive value $92 \%$ in the prediction of abnormal outcomes. So, again proving that normal CTG is more predictive of normal outcomes than abnormal CTG regarding abnormal outcomes. ${ }^{11}$

Limitations of our study include that it was conducted in a tertiary care teaching institute, which might not reflect the true study population, technical and clinical expertise.

\section{CONCLUSIONS}

CTG should only be used as a screening tool for monitoring of foetal status during labour. A suspicious CTG should be followed up or confirmed with a better diagnostic test. Whereas a pathological CTG is highly predictive of poor perinatal outcome, warranting immediate intervention. Moreover it is worth remembering that normal CTG is more predictive of normal outcomes than abnormal CTG regarding abnormal outcomes.

\section{ACKNOWLEDGEMENTS}

We are grateful to Dr Lavanya Rai, Dr Pratap Kumar, Dr Muralidhar V Pai, Dr Jyothi Shetty and Dr Sapna V Amin for allowing us to include their patients for our study.

\author{
Funding: No funding sources \\ Conflict of interest: None declared \\ Ethical approval: The study was approved by the \\ Institutional Ethics Committee
}




\section{REFERENCES}

1. Hon EH. The electronic evaluation of FHR: preliminary report. Am J Obstet Gynecol. 1958;75:1215-30.

2. Hon EH, Hess OW. The clinical value of ECG. Am J Obstet Gyneco. 1960;79:1012-23.

3. Banta HD, Thacker SB. Fetal monitoring. Obstet Gynecol. 1979;54:667-70.

4. Stanton CK, Holtz SA. Studies in Family Planning. 2006;37:41.

5. Barber EL, Lundsberg LS, Belanger K, Pettker CM, Funai EF, Illuzzi JL. Indications contributing to the increasing cesarean delivery rate. Obstet Gynecol. 2011;118(1):29-38.

6. Alfirevic Z, Devane D, Gyte GM. Continuous cardiotocography as a form of electronic fetal monitoring for fetal assessment during labour. Cochrane Database Syst Rev 3:CD006066, 2006.

7. Grant A, O'Brien N, Joy MT. Cerebral palsy among children born during the Dublin randomised trial of intrapartum monitoring. Lancet. 1989;2:1233-6.
8. Grivell RM, Alfirevic Z, Gyte GM, Devane D. Antenatal cardiotocography for fetal assessment. Cochrane Database Syst Rev 12:CD007863, 2012.

9. Ropacka-Lesiak M, Korbelak T, Breborowicz G. Hypoxia index in the prediction of abnormal CTG at delivery in uncomplicated pregnancies. Neuro Endocrinol Lett. 2013;34(1):75-80.

10. Tasnim N, Mahmud G, Akram S. Predictive accuracy of intrapartum cardiotocography in terms of fetal acid base status at birth. J Coll Physicians Surg Pak. 2009;19(10):632-5.

11. Sultana J, Chowdhury TA, Begum K, Khan MH. Comparison of normal and abnormal cardiotocography with pregnancy outcomes and early neonatal outcomes. Mymensingh Med J. 2009;18(1 Suppl):S103-7.

Cite this article as: Xavier AA, Pandey D, Dogra L, Lewis LE. Cardiotocography in a perinatal armamentarium: boon or bane?. Int J Reprod Contracept Obstet Gynecol 2015;4:2000-4. 